\title{
Humoral immune consequences of Staphylococcus aureus ST239-associated bacteremia
}

\author{
H. Ghasemzadeh-Moghaddam ${ }^{1,2}$ • WJB van Wamel $^{3}$ • A. van Belkum ${ }^{3,4}$ • R. A. Hamat ${ }^{1}$. \\ M. Tavakol ${ }^{3}$ V. K. Neela ${ }^{1}$ (D)
}

Received: 12 October 2017 / Accepted: 16 October 2017 /Published online: 4 November 2017

(C) Springer-Verlag GmbH Germany 2017

\begin{abstract}
The humoral immune responses against 46 different staphylococcal antigens in 27 bacteremia patients infected by clonally related methicillin-resistant Staphylococcus aureus (MRSA) strains of a single sequence type (ST) 239 were investigated. A group of non-infected patients $(n=31)$ hospitalized for different reasons served as controls. All strains were confirmed as ST 239 by $S$. aureus and $m e c A$-specific PCR, spa, and multi-locus sequence typing (MLST). In each bacteremia patient, a unique pattern of S. aureus antigen-specific immune responses after infection was observed. Antibody levels among bacteremia patients were significantly higher than controls for $\operatorname{HlgB}(P=0.001)$, LukD $(P=0.009)$, LukF $(P=0.0001)$, SEA $(P=0.0001)$, SEB $(P=0.011)$, SEC $(P=0.010)$, SEQ $(P=0.049)$, IsaA $(P=0.043)$, IsdA $(P=0.038), \operatorname{IsdH}(P=0.01), \operatorname{SdrD}$ $(P=0.001), \operatorname{SdrE}(P=0.046)$, EsxA $(P=0.0001)$, and SA0104 $(P=0.0001)$. On the other hand, the antibody levels were significantly higher among controls for SSL3 $(P=0.009)$, SSL9 $(P=0.002)$, and SSL10 $(P=0.007)$ when the IgG level on the day of infection was compared with that
\end{abstract}

Electronic supplementary material The online version of this article (https://doi.org/10.1007/s10096-017-3124-3) contains supplementary material, which is available to authorized users

\section{K. Neela}

vasantha@upm.edu.my

1 Department of Medical Microbiology and Parasitology, Faculty of Medicine and Health Sciences, Universiti Putra Malaysia, 43400 Serdang, Selangor, Malaysia

2 Faculty of Medicine, Natural Products and Medicinal Plants Research Center, North Khorasan University of Medical Sciences, Bojnurd, Iran

3 Erasmus Medical Centre Rotterdam, Rotterdam, The Netherlands

4 bioMérieux, Data Analytics Unit, La Balme les Grottes, France measured on the day of admission. Diversity was observed in the immune response against the antigens. However, a set of antigens (IsaA, IsdA, IsdH, SdrD, and $\mathrm{HlgB}$ ) triggered a similar type of immune response in different individuals. We suggest that these antigens could be considered when developing a multi-component (passive) vaccine. SEA and/or its specific antibodies seem to play a critical role during ST239 MRSA bacteremia and SEA-targeted therapy may be a strategy to be considered.

\section{Introduction}

Staphylococcus aureus is a well-recognized nosocomial pathogen. Nosocomial methicillin-resistant $S$. aureus (MRSA) infections increase hospital stay, antibiotic use, overall hospitalization costs, morbidity, and mortality [1-3]. Among all bacterial infections, mortality due to MRSA remains the most frequent. The two main factors that render $S$. aureus a successful nosocomial pathogen are its frequent and multiple drugresistant properties and its multi-virulence. The declining trend observed in the development of new antibiotics is likely due to huge costs and the lack of significant returns on investment owing to rapidly emerging resistance. Producing protective immunity against $S$. aureus was and still is important, but using live bacterial cells or killed bacteria has not yet been successful [4]. This requires alternative approaches, including the prophylactic or therapeutic use of antibodies.

Many geographical regions harbor selective and local epidemic MRSA strains. Several large-scale epidemiological investigations demonstrated that most hospital-acquired MRSA infections are caused by a relatively small number of MRSA clones spread worldwide. The most frequently encountered clones are the Iberian (ST247-IA), Brazilian/Hungarian (ST239-III), Berlin (ST45-IV), New York/Japan (ST5-II), 
pediatric (ST5-IV), EMRSA-15 (ST22-IV), and EMRSA-16 (ST36-II) clones [5]. For example, all strains from Korea and Japan belong to clonal complex 5 (CC5) whereas strains from the rest of the Asian countries are primarily CC239 [6]. ST239 is the most successful clone isolated from hospitalized patients in Malaysia [7, 8].

Different staphylococcal determinants, such as purified capsular polysaccharide (types 5 and 8 ) or individual surface proteins, e.g., clumping factor A (ClfA) [9], clumping factor $\mathrm{B}$ (ClfB) [10], iron-regulated surface determinant $\mathrm{B}$ (IsdB) [11], or fibronectin-binding protein (FnBP) [12] only generated immune responses that gave partial protection against $S$. aureus in experimental animals [13], whereas vaccination in humans failed [14-16]. A successful vaccine candidate should be immunogenic and should play an essential role in staphylococcal survival. Failure of human experiments indicates that animal models are not always good mimics for the human body. In addition, there is a gap in the knowledge of in vivo expression of virulence factors of $S$. aureus, emphasizing the need to better understand the role and expression status of $S$. aureus virulence factors in humans [17]. Transcriptomics of MRSA USA300 cultured in human blood or serum revealed up-regulation of only a limited number of genes encoding putative $S$. aureus virulence factors [18]. Genes encoding the cytolytic toxins $\mathrm{HlgA}, \mathrm{HlgB}$, and $\mathrm{HlgC}$ were among the most frequently upregulated genes at various time points [18].

Studies on antibody repertoires against $S$. aureus antigens among healthy blood donors and infected patients revealed marked differences, whereas a noticeable heterogeneity was observed between given individuals [19]. More recent studies revealed that patients infected with $S$. aureus had clearly different, individualized humoral responses [20,21], but no clear association among specific infection and humoral response against one or more virulence factors was documented. Sera from patients with $S$. aureus blood infection were examined; antibody levels increased against immune modulatory proteins and MSCRAMMs such as iron-regulated surface determinant A (IsdA) in most cases [20]. However, the situation was different in other sets of bacteremia patients, where antibodies against a putative iron-regulated $\mathrm{ABC}$ transporter, SA0688, showed the highest serum levels after challenge [17].

$S$. aureus strains responsible for human infections have clearly different genetic backgrounds with variable core genome sequences [22]. Most of these differences cover genes related to virulence located on pathogenicity island or other mobile genetic elements [17]. Hence, most pathogenic $S$. aureus strains share a vast arsenal of virulence factors. It could very well be that the diversity found in antistaphylococcal humoral immune response is the result of staphylococcal genetic diversity. This indicates that different $S$. aureus strains express different spectra of virulence factors during infection. On the other hand, the ability of different individuals to mount a humoral response against specific antigens could be different as well.

We tried to come to a better understanding of the human immune responses against $S$. aureus virulence factors encoded by a single dominant sequence type (ST) in different infected and non-infected individuals. In the present study, IgG antibody levels against an array of staphylococcal antigens was compared in bacteremia patients infected by ST239 S. aureus strains.

\section{Materials and methods}

\section{Study cases}

The study involved patients admitted to Hospital Kuala Lumpur (HKL) from the 19 September 2011 to the 14 February 2012, who developed confirmed MRSA bacteremia during their hospital stay. Two serum samples were collected from each patient. The first blood sample was taken on the day of onset of infection whereas a second sampling was performed 7 days later. Thirty-six adult patients were included in the study; at the end, 27 patients remained. Nine patients were excluded as $8(21 \%)$ passed away during the first week of infection before a second blood sample could be collected and 1 patient was infected with a genetically different MRSA strain. The mean age of the patients was 52.8 years (range 1677 years) and $70.3 \%$ were male. According to the three major ethnic groups in Malaysia, the study population comprised Malay $(n=13 ; 48.1 \%)$, Chinese $(n=7 ; 25.9 \%)$, and Indians $(n=7 ; 25.9 \%)$. All patients were treated with antibiotics according to hospital guidelines.

A group of hospitalized patients $(n=31)$ in HKL without staphylococcal infection during hospitalization served as controls, where serum samples on admission day and day 7 after admission were collected. The mean age for the control group was 46.2 (range $27-70$ years), $70.9 \%$ of whom were male.

All patients and controls were subjected to nasal swab culture to determine their staphylococcal carrier status. A sterile transwab (Medical Wire, UK) was rotated four times in both anterior nasal vestibules and cultured on mannitol salt agar and chrome agar medium (BD Diagnostics, Heidelberg, Germany).

The study was performed with the approval of the Universiti Putra Malaysia ethics committee according to the rules of good clinical practice and the Malaysian statutory definition of clinical trials. Patients received verbal and written information from nursing personnel. Patients or relatives provided verbal consent, which was noted in the nursery record. Patients had to be mentally and physically capable of taking the decision to participate in the study. For patients unable to make such a decision, guardians were informed and their initial agreement was obtained. Before official 
participation, either the patients or their guardians had to provide official written consent.

\section{Bacterial isolation and growth conditions}

Blood samples were cultured using the Becton Dickinson blood culture system (BD BACTEC ${ }^{\mathrm{TM}}$ ). All Gram-positive cocci resembling staphylococci were subjected to catalase testing, coagulase tube testing, and Sa442 PCR for definitive species confirmation [23]. MRSA screening was performed by a cefoxitin disk diffusion test on a Mueller Hinton agar plate for all isolates. Whenever resistance to cefoxitin was observed, MRSA status was confirmed by mecA gene PCR [5].

\section{Genotyping and virulence gene profiles of $S$. aureus isolates}

For genotyping, chromosomal DNA was extracted using the DNeasy Kit (Qiagen). Staphylococcal protein A (spa) typing and multi-locus sequence typing (MLST) was performed as described earlier [24, 25]. Sequence types (STs) and clonal clusters (CC) were assigned using the $S$. aureus MLST database (www.mlst.net) hosted by Imperial College in London, UK. SCCmec typing of MRSA strains was performed as described before [7, 26].

For all 27 patients with staphylococcal bacteremia, $S$. aureus strains isolated from blood were screened for the presence of 46 genes encoding different antigens by PCR [17].

\section{Antigens}

All 6X His-tagged recombinant protein antigens were coupled with $\mathrm{xMAP}{ }^{\circledR}$ beads (Luminex Corporation, Austin, TX, USA) for fluorescence-activated cell sorting (FACS) using Luminex experiments. The antigens investigated in the present study include: protein secretion system ESX-1-associated factors EsxA and EsxB; nuclease (Nuc); peptidoglycan hydrolase LytM; immune-dominant antigen A (IsaA); glucosaminidase; lipase; foldase-protein PrsA; clumping factor A and B (ClfA and ClfB); SD-repeat containing proteins D and $\mathrm{E}$ (SdrD and $\mathrm{SdrE}$ ); iron-responsive surface determinants $\mathrm{A}$ and $\mathrm{H}$ (IsdA and IsdH); fibronectin-binding proteins $\mathrm{A}$ and B (FnbpA and FnbpB); extracellular fibrinogen-binding protein (Efb); S. aureus surface protein G (SasG); staphylococcal complement inhibitor (SCIN); chemotaxis inhibitory protein of $S$. aureus (CHIPS); formyl peptide receptor-like inhibitory protein (FLIPr); formyl peptide receptor-like inhibitory like protein (FLIPr-L); alpha toxin; gamma-hemolysin B (HlgB); leukocidins D and F (LukD, LukF); staphylococcal enterotoxins A-E, G-J, M-O, Q, R (SEA-SEE, SEG-SEJ, SEMSEO, SEQ, SER); exfoliative toxins A (ETA); toxic shock syndrome toxin 1 (TSST-1); staphylococcal superantigen- like proteins $1,3,5,9,10$, and 11 (SSL1, SSL3, SSL5, SSL9, SSL10, and SSL11); and hypothetical proteins (SA0104 and SA2097).

SasG, SdrD, SdrE, ClfB, IsdA, IsdH, FnbpA, and FnbpB were purified as described previously [21, 27]. The genetic constructs were kindly provided by T. Foster (Trinity College, Dublin, Ireland). Alpha toxin, HlgB, LukD, LukE, LukF, LukS, SEA, and SEC were prepared as described previously [17]. All other proteins were kindly provided by other research groups, as indicated in the acknowledgments. Coupling protein antigens with XMAP ${ }^{\circledR}$ beads was performed using a protocol described earlier [28, 29].

\section{Measurement of antibodies against $S$. aureus antigens}

Fluorescence-activated cell sorting (xMAP®; Luminex Corporation) was used for measuring antibodies against staphylococcal antigens in serum samples of bacteremia patients, as described before [20, 28, 30]. Duplicate measurements were performed for all serum samples diluted 100 times in PBS. A 200 times-diluted secondary phycoerythrin (PE)-labeled goat anti-human antibody against total IgG was used for the detection of antibody binding. Median fluorescence intensities (MFIs), a semi-quantitative measure of antibody levels, were averaged. All duplicated measurements that had a coefficient of variation $(\mathrm{CV})$ of more than $25 \%$ were excluded from statistical analysis.

\section{Statistics}

The (fold) increase or decrease in the MFI values for each antigen on day 1 and day 7 among patients and controls were calculated. The nonparametric Mann-Whitney $U$ test was used to compare the antibody levels of bacteremia patients and controls, and the nonparametric Wilcoxon signed ranks test was used to compare the antibody levels of bacteremia patients and controls on days 1 and 7. All statistical analyses were performed using SPSS version 15.0 and $P$ values less than 0.05 were considered significant.

\section{Results}

Thirty-seven $S$. aureus strains were isolated from 27 bacteremia patients (10 from anterior nares and 27 from the blood stream). Among the nasal isolates, 8 were typed as t037/ST239 whereas the other two were typed as t421/ST239, and t6811/non-typeable by MLST. One blood isolate was typed as t421/ST239 whereas all others were t037/ST239. All carrier patients showed nondiscriminate types for the isolates from blood and nares (Table 1). Among controls, 8 were MSSA nasal carrier with bacteria showing genetically different backgrounds (t8021/NT, 
Table 1 Patients' demographic data and strain typing results. First blood was taken after confirmation of bacteremia in the patient

\begin{tabular}{|c|c|c|c|c|c|c|c|c|c|c|c|}
\hline $\begin{array}{l}\text { Patient } \\
\text { ID }\end{array}$ & $\begin{array}{l}\text { Carrier } \\
\text { status }\end{array}$ & Race & Age & $\begin{array}{l}\text { Date } \\
\text { blood } \\
\text { culture } \\
\text { taken }\end{array}$ & $\begin{array}{l}\text { Bacteremia } \\
\text { confirmation } \\
\text { date }\end{array}$ & $\begin{array}{l}\text { First } \\
\text { blood } \\
\text { taken } \\
\text { date }\end{array}$ & $\begin{array}{l}\text { Second } \\
\text { blood } \\
\text { taken } \\
\text { date }\end{array}$ & $\begin{array}{l}\text { Blood- } \\
\text { isolated strain } \\
\text { spa type } \\
\text { (MLST) }\end{array}$ & $\begin{array}{l}\text { Nasally } \\
\text { isolated strain } \\
\text { spa type } \\
\text { (MLST) }\end{array}$ & Ward & $\begin{array}{l}\text { Reason for } \\
\text { hospitalization }\end{array}$ \\
\hline $\mathrm{P} 1$ & \multirow[t]{17}{*}{$\begin{array}{r}\text { Noncarrier } \\
\text { patients }\end{array}$} & Chinese & 65 & 18.9.11 & 21.9.11 & 19.9.11 & 22.9.11 & t037 (ST239) & - & $\mathrm{U} 2 \mathrm{~B}$ & $\begin{array}{l}\text { ESRF on } \\
\text { dialysis }\end{array}$ \\
\hline $\mathrm{P} 2$ & & Malay & 74 & 20.9.11 & 22.9 .11 & 21.9.11 & 26.9.11 & t037 (ST239) & - & SW14 & $\begin{array}{l}\text { Lung } \\
\text { infection, } \\
\text { sepsis }\end{array}$ \\
\hline P4 & & Malay & 57.8 & 1.10 .11 & 4.10 .11 & 4.10 .11 & 7.10 .11 & t037 (ST239) & - & SW27 & $\begin{array}{l}\text { Line-related } \\
\text { sepsis }\end{array}$ \\
\hline P5 & & Malay & 55.8 & 11.10 .11 & 13.10 .11 & 14.10 .11 & 19.10.11 & t037 (ST239) & - & SW13 & Wound \\
\hline P6 & & Malay & 23.2 & 14.10 .11 & 16.10 .11 & 17.10 .11 & 20.10 .11 & t037 (ST239) & - & NW3 & Burn \\
\hline P9 & & Indian & 22 & 21.10 .11 & 23.10 .11 & 22.10 .11 & 27.10 .11 & t037 (ST239) & - & NW3 & Burn \\
\hline $\mathrm{P} 10$ & & Chinese & 71 & 25.10 .11 & 26.10 .11 & 27.10 .11 & 1.11 .11 & t037 (ST239) & - & SW6 & Wound \\
\hline P15 & & Malay & 55 & 27.10 .11 & 29.10 .11 & 30.10 .11 & 2.12 .11 & t037 (ST239) & - & SW13 & Wound \\
\hline P17 & & Chinese & 81 & 17.11.11 & 18.11.11 & 18.11.11 & 22.11 .11 & t037 (ST239) & - & SW13 & Wound \\
\hline P19 & & Indian & 60.6 & 6.12 .11 & 7.12 .11 & 7.12 .11 & 10.12 .11 & t037 (ST239) & - & SW26 & Wound \\
\hline P22 & & Indian & 46.2 & 4.1.12 & 5.1 .12 & 5.1 .12 & 10.1 .12 & t037 (ST239) & - & $\mathrm{U} 2 \mathrm{~B}$ & $\begin{array}{l}\text { Dialysis, renal } \\
\text { failure }\end{array}$ \\
\hline $\mathrm{P} 23$ & & Malay & 45 & 4.1 .12 & 5.1 .12 & 5.1 .12 & 9.1 .12 & t037 (ST239) & - & SW26 & Unknown \\
\hline P25 & & Malay & 77 & 11.1 .12 & 13.1.12 & 11.1.12 & 16.1.12 & t037 (ST239) & - & SW27 & Wound, ESRF \\
\hline P26 & & Malay & 55 & 17.1.12 & 18.1.12 & 17.1.12 & 27.1.12 & t037 (ST239) & - & GICU.NW10 & $\begin{array}{l}\text { Sacral sore } \\
\text { grade } 2\end{array}$ \\
\hline P27 & & Malay & 20.2 & 20.1 .12 & 21.1.12 & 20.1 .12 & 26.1 .12 & t037 (ST239) & - & NW11 & Unknown \\
\hline P28 & & Malay & 16.3 & 27.1.12 & 28.1 .12 & 27.1.12 & 1.2 .12 & t037 (ST239) & - & Burn & Burn \\
\hline P31 & & Malay & 62.5 & 12.2 .12 & 13.2.12 & 14.2.12 & 17.2.12 & t037 (ST239) & - & $\mathrm{U} 2 \mathrm{~B}$ & $\begin{array}{l}\text { Dialysis, renal } \\
\text { failure }\end{array}$ \\
\hline P3 & \multirow{10}{*}{$\begin{array}{l}\text { Carrier } \\
\text { patients }\end{array}$} & Indian & 69.2 & 24.9.11 & 26.9 .11 & 25.9.11 & 2.10 .11 & t037 (ST239) & t037 (ST239) & NW23 & Sepsis \\
\hline P8 & & Chinese & 45 & 18.10 .11 & 20.10 .11 & 21.10 .11 & 24.10 .11 & t037 (ST239) & t037 (ST239) & UN2B & ESRF \\
\hline P11 & & Indian & 57.9 & 29.10 .11 & 30.10 .11 & 31.10 .11 & 3.11 .11 & t037 (ST239) & t037 (ST239) & U1B & $\begin{array}{l}\text { Dialysis, renal } \\
\text { failure }\end{array}$ \\
\hline $\mathrm{P} 12$ & & Chinese & 25.7 & 30.10 .11 & 1.11 .11 & 3.11 .11 & 8.11 .11 & t421(ST239) & t421(ST239) & UN1B & $\begin{array}{l}\text { Dialysis, renal } \\
\text { failure }\end{array}$ \\
\hline P13 & & Chinese & 81 & 3.11 .11 & 5.11 .11 & 4.11.11 & 8.11 .11 & t037 (ST239) & t6811(NT) & NW2 & Unknown \\
\hline P14 & & Chinese & 48 & 29.10 .11 & 31.11 .11 & 29.10 .11 & 9.11 .11 & t037 (ST239) & t037 (ST239) & UN2B & $\begin{array}{l}\text { Dialysis, renal } \\
\text { failure }\end{array}$ \\
\hline P16 & & Indian & 67 & 22.11 .11 & 24.11 .11 & 23.11 .11 & 27.11 .11 & t037 (ST239) & t037 (ST239) & GICU & Unknown \\
\hline P18 & & Malay & 65 & 4.12 .11 & 6.12 .11 & 5.12 .11 & 9.12 .11 & t037 (ST239) & t037 (ST239) & UN2A & $\begin{array}{l}\text { Dialysis, renal } \\
\text { failure }\end{array}$ \\
\hline $\mathrm{P} 21$ & & Malay & 65.1 & 3.1 .12 & 4.1 .12 & 4.1.12 & 11.1.12 & t037 (ST239) & t037 (ST239) & $\mathrm{U} 2 \mathrm{~A}$ & $\begin{array}{l}\text { Dialysis, renal } \\
\text { failure }\end{array}$ \\
\hline P24 & & Indian & 61 & 9.1 .12 & 10.1 .12 & 10.1 .12 & 14.1 .12 & t037 (ST239) & t037 (ST239) & GICU.NW10 & Unknown \\
\hline
\end{tabular}

$E S R F$ end-stage renal failure, GICU general intensive care unit, $U$ and $U N$ urology wards, $S W$ surgery ward, $N W$ general medicine, $N T$ nontypeable

t189/ST188 $[n=2]$, t735/ST97, t03/ST1153, t10560/ST96, t1445/ST1209, 127/ST10).

\section{Polymer chain reaction}

Genes for staphylococcal super antigen-like proteins (SSL1, SSL3, SSL5, SSL9, SSL10, SSL11), alpha toxin, clumping factor A and B, fnbpA, fnbpB, Efb, immune-dominant staphylococcal antigen (IsaA), Iron-regulated surface determinant proteins (IsdA, IsdH), PrsA, sasG, serine-aspartate (SD) repeat-containing proteins (sdrD, sdrE), SEA, SEJ, SEQ, SEM, leukocidin D (LUKD), chemotaxis inhibitory protein of staphylococci (CHIPS), EsxA, HlgB, FlipR, FlipR-L, and lipase were observed in all ST239 strains tested, whereas other 
genes were not detected (Table 2, Supplementary Tables 1, 2). The ST239 strains could not be discriminated by this type of PCR testing, as the results for all genes were equal for all isolates.

\section{MFI values on day 1 and day 7 among patients}

Mean MFI values reflecting IgG antibody levels against the corresponding antigens are illustrated in Table 2 and Supplementary Tables 1 and 2. MFIs measured on day 1 and day 7, when compared with the individual patients and controls, showed no significant differences for most antigens (Supplementary Tables 1, 2).

However, the mean MFI was significantly higher on day 7 for ClfA $(P=0.001)$, FnbpA $(P=0.020)$, sasG $(P=0.015)$, SEH $(P=0.037)$, SA0104 $(P=0.045)$, and SA2097 $(P=0.011)$ in the control group. For the patient group a higher MFI was observed for ClfA $(P=0.02)$, FnbpA $(P=0.008)$, IsaA $(P=0.004)$, and SER $(P=0.024$; Table 2 , Supplementary Tables 1,2).

\section{MFI values among patients and controls}

In each bacteremia patient a unique pattern of $S$. aureus-specific antibody levels after infection were observed (Supplementary Figs. 1-16). The median MFI among patients increased compared with controls for most of the antigens. Antibody levels among bacteremia patients were significantly higher for the following antigens: $\mathrm{HlgB}(P=0.001), \operatorname{LukD}$ $(P=0.009)$, LukF $(P=0.0001)$, SEA $(P=0.0001)$, SEB $(P=0.011)$, SEC $(P=0.010)$, SEQ $(P=0.049)$, IsaA $(P=0.043), \operatorname{IsdA}(P=0.038), \operatorname{IsdH}(P=0.01), \operatorname{SdrD}$ $(P=0.001), \operatorname{SdrE}(P=0.046)$, EsxA $(P=0.0001)$, SA2097 $(P=0.001)$, and SA0104 $(P=0.0001)$ when MFIs on the day of infection (day 1) were compared with day 1 in the control group (Table 2, Supplementary Tables 1, 2; Supplementary Figs. 1-16).

When MFI on day 7 after infection was compared with those of controls, antibody levels were still significantly higher among bacteremia patients for most of the abovementioned antigens except for SEB $(P=0.057)$, SEQ $(P=0.061)$, and $\operatorname{SdrE}(P=0.387$; Table 2 , Supplementary Tables 1, 2). However, for certain antigens, such as SCIN $(P=0.046)$, SEI $(P=0.038)$, and EAP $(P=0.047$; Table 2 , Supplementary Tables 1,2$)$, the MFI value was significantly higher only on day 7 after infection.

On the other hand, for SSL3 $(P=0.009, P=0.002)$, SSL9 $(P=0.002, P=0.0001)$, and SSL10 $(P=0.007, P=0.001)$, significantly lower MFIs were detected on day 1 and day 7 of infection. For lipase $(P=0.001)$, the MFIs measured for patients were lower after 7 days (Table 2, Supplementary Tables 1, 2).

\section{Discussion}

In the present study, we investigated the humoral immune response against 46 staphylococcal antigens in bacteremia patients, all infected by a similar MRSA type (ST239), versus controls. The presence of the genes corresponding to the antigens was also investigated. The observation of unique patterns of $S$. aureus-specific immune responses in each patient is in concordance with data reported by Verkaik et al. (2010) [20]. The genetic diversity among the strains and expression of different staphylococcal proteins during infection or host factors were suggested as possible causes for this variability [20]. In the current study, all bacteremia patients were infected by highly homologous MRSA ST239 strains. Therefore, differences in the level of specific IgG antibodies between some patients here could be due to the difference in the level of immunity among patients as a result of various past exposures.

We observed significant increases in the antibody levels against leukocidin F, whereas the PCR result for the corresponding genes was negative in ST239 MRSA strains. Such an increased response against certain antigens in the absence of the corresponding genes has been reported before [20] and could be due to cross-reactivity with different antigenic components with structurally similar domains [31-33]. Den Reijer and colleagues reported the immunological cross-reactivity between leukocidins F and D and HlgB [17]. Interestingly, genes for leukocidin D and HlgB were present in the genomes of the ST239 MRSA strains involved and IgG antibody levels against these toxins were significantly higher among bacteremia patients than among controls. Cross-reactivity may also explain the higher MFI levels against SEB and SEC among patients, again in the absence of the corresponding genes, whereas clearly higher levels of antibodies against the structurally similar enterotoxins (SEA and SEQ) were detected.

We observed significantly higher levels of IgG antibody against $\mathrm{HlgB}$ in bacteremia patients. Recently, Malachowa et al. showed the up-regulation of $h l g A B C$ in USA300 during culture in human blood in combination with the induction of in vitro lysis of human PMNs [18]. Similar findings were also presented by den Reijer et al. in two distinct $S$. aureus strains (t209, t122), where the IgG-binding protein $s b i$ and the gamma-hemolysin A and B precursors were up-regulated when strains were cultured in blood [17]. Our data, together with those of the above studies, suggest that HlgB could be a potential vaccine candidate, as it seems to trigger an immune response irrespective of the strain type.

The multi-virulent nature of $S$. aureus and the fact that monoclonal vaccines failed emphasize the importance of multi-component vaccines [34]. A combined vaccine of four cell-wall-anchored proteins (IsdA, IsdH, SdrD, and SdrE) showed successful protection in an animal model [13]. On the other hand, a recent study on IsaA, which is a secreted protein with an ill-defined housekeeping function, put it 
Table 2 Luminex assay and statistical analysis

\begin{tabular}{|c|c|c|c|c|c|c|c|c|c|}
\hline \multirow[t]{2}{*}{ Antigen } & \multirow[t]{2}{*}{ PCR } & \multirow[t]{2}{*}{$\begin{array}{l}\text { Study } \\
\text { group }\end{array}$} & \multirow[t]{2}{*}{$\begin{array}{l}\text { Mean } \\
\text { MFI }\end{array}$} & $\begin{array}{l}\text { Mean } \\
\text { MFI }\end{array}$ & \multirow[t]{2}{*}{$\begin{array}{l}\text { MFI on day } 1 \\
\text { compared } \\
\text { with day } 7 \\
\text { group }\left(P^{*}\right)\end{array}$} & Patient: & control & \multicolumn{2}{|c|}{$\begin{array}{l}\text { MFI among } \\
\text { patients in } \\
\text { comparison to } \\
\text { controls }\left(P^{* *}\right)\end{array}$} \\
\hline & & & & Day7 & & Day 1 & Day 7 & $\begin{array}{l}\text { Day } \\
1\end{array}$ & Day7 \\
\hline HIgb & Positive & $\begin{array}{l}\text { Patients } \\
\text { Controls }\end{array}$ & $\begin{array}{l}15,379 \\
12,552\end{array}$ & $\begin{array}{l}15,622 \\
12,481\end{array}$ & $\begin{array}{l}0.400 \\
0.800\end{array}$ & $1.23: 1$ & $1.25: 1$ & 0.001 & 0.000 \\
\hline LUKD & Positive & $\begin{array}{l}\text { Patients } \\
\text { Controls }\end{array}$ & $\begin{array}{l}15,413 \\
13,084\end{array}$ & $\begin{array}{l}15,630 \\
13,074\end{array}$ & $\begin{array}{l}0.486 \\
0.936\end{array}$ & $1.18: 1$ & $1.20: 1$ & 0.009 & 0.005 \\
\hline LUKF & Negative & $\begin{array}{l}\text { Patients } \\
\text { Controls }\end{array}$ & $\begin{array}{l}4,748 \\
2,905\end{array}$ & $\begin{array}{l}5,168 \\
3,016\end{array}$ & $\begin{array}{l}0.137 \\
0.189\end{array}$ & $1.63: 1$ & $1.71: 1$ & 0.000 & 0.000 \\
\hline IsdA & Positive & $\begin{array}{l}\text { Patients } \\
\text { Controls }\end{array}$ & $\begin{array}{l}6,169 \\
4,403\end{array}$ & $\begin{array}{l}6,461 \\
4,663\end{array}$ & $\begin{array}{l}0.130 \\
0.658\end{array}$ & $1.40: 1$ & $1.39: 1$ & 0.038 & 0.046 \\
\hline IsdH & Positive & $\begin{array}{l}\text { Patients } \\
\text { Controls }\end{array}$ & $\begin{array}{l}4,118 \\
1,038\end{array}$ & $\begin{array}{l}4,185 \\
1,172\end{array}$ & $\begin{array}{l}0.683 \\
0.060\end{array}$ & $3.97: 1$ & $3.57: 1$ & 0.010 & 0.016 \\
\hline IsaA & Positive & $\begin{array}{l}\text { Patients } \\
\text { Controls }\end{array}$ & $\begin{array}{l}9,912 \\
6,544\end{array}$ & $\begin{array}{l}11,010 \\
6,979\end{array}$ & $\begin{array}{l}0.004 \\
0.204\end{array}$ & $1.51: 1$ & $1.58: 1$ & 0.043 & 0.010 \\
\hline $\mathrm{SdrD}$ & Positive & $\begin{array}{l}\text { Patients } \\
\text { Controls }\end{array}$ & $\begin{array}{l}486 \\
137\end{array}$ & $\begin{array}{l}765 \\
154\end{array}$ & $\begin{array}{l}0.209 \\
0.043\end{array}$ & $3.54: 1$ & $4.97: 1$ & 0.001 & 0.005 \\
\hline SEA & Positive & $\begin{array}{l}\text { Patients } \\
\text { Controls }\end{array}$ & $\begin{array}{l}14,726 \\
5,443\end{array}$ & $\begin{array}{l}15,257 \\
5,540\end{array}$ & $\begin{array}{l}0.101 \\
0.491\end{array}$ & $2.71: 1$ & $2.75: 1$ & 0.000 & 0.000 \\
\hline SEC & Negative & $\begin{array}{l}\text { Patients } \\
\text { Controls }\end{array}$ & $\begin{array}{l}13,307 \\
10,690\end{array}$ & $\begin{array}{l}13,470 \\
11,501\end{array}$ & $\begin{array}{l}0.683 \\
0.280\end{array}$ & $1.24: 1$ & $1.17: 1$ & 0.010 & 0.040 \\
\hline SSL3 & Positive & $\begin{array}{l}\text { Patients } \\
\text { Controls }\end{array}$ & $\begin{array}{l}6,079 \\
8,613\end{array}$ & $\begin{array}{l}5,939 \\
8,950\end{array}$ & $\begin{array}{l}0.773 \\
0.261\end{array}$ & $1: 1.42$ & $1: 1.51$ & 0.009 & 0.002 \\
\hline SSL9 & Positive & $\begin{array}{l}\text { Patients } \\
\text { Controls }\end{array}$ & $\begin{array}{l}3,583 \\
5,838\end{array}$ & $\begin{array}{l}3,460 \\
6,124\end{array}$ & $\begin{array}{l}0.564 \\
0.237\end{array}$ & $1: 1.63$ & $1: 1.77$ & 0.002 & 0.000 \\
\hline SSL10 & Positive & $\begin{array}{l}\text { Patients } \\
\text { Controls }\end{array}$ & $\begin{array}{l}5,454 \\
8,014\end{array}$ & $\begin{array}{l}5,319 \\
8,346\end{array}$ & $\begin{array}{l}0.269 \\
0.106\end{array}$ & $1: 1.47$ & $1: 1.57$ & 0.007 & 0.001 \\
\hline EsxA & Positive & $\begin{array}{l}\text { Patients } \\
\text { Controls }\end{array}$ & $\begin{array}{l}29 \\
13\end{array}$ & $\begin{array}{l}26 \\
12\end{array}$ & $\begin{array}{l}0.927 \\
0.367\end{array}$ & $2.22: 1$ & $2.21: 1$ & 0.000 & 0.000 \\
\hline SA0104 & Negative & $\begin{array}{l}\text { Patients } \\
\text { Controls }\end{array}$ & $\begin{array}{l}296 \\
50\end{array}$ & $\begin{array}{l}326 \\
57\end{array}$ & $\begin{array}{l}0.304 \\
0.045\end{array}$ & $5.93: 1$ & $5.72: 1$ & 0.000 & 0.000 \\
\hline IsdA & Positive & $\begin{array}{l}\text { Patients } \\
\text { Controls }\end{array}$ & $\begin{array}{l}6,169 \\
4,403\end{array}$ & $\begin{array}{l}6,461 \\
4,663\end{array}$ & $\begin{array}{l}0.130 \\
0.658\end{array}$ & $1.40: 1$ & $1.39: 1$ & 0.038 & 0.046 \\
\hline IsdH & Positive & $\begin{array}{l}\text { Patients } \\
\text { Controls }\end{array}$ & $\begin{array}{l}4,118 \\
1,038\end{array}$ & $\begin{array}{l}4,185 \\
1,172\end{array}$ & $\begin{array}{l}0.683 \\
0.060\end{array}$ & $3.97: 1$ & $3.57: 1$ & 0.010 & 0.016 \\
\hline SA2097 & Negative & $\begin{array}{l}\text { Patients } \\
\text { control }\end{array}$ & $\begin{array}{l}49 \\
16\end{array}$ & $\begin{array}{l}80 \\
21\end{array}$ & $\begin{array}{l}0.134 \\
0.011\end{array}$ & $3.08: 1$ & $3.81: 1$ & 0.001 & 0.001 \\
\hline IsaA & Positive & $\begin{array}{l}\text { Patients } \\
\text { Controls }\end{array}$ & $\begin{array}{l}9,912 \\
6,544\end{array}$ & $\begin{array}{l}11,010 \\
6,979\end{array}$ & $\begin{array}{l}0.004 \\
0.204\end{array}$ & $1.51: 1$ & $1.58: 1$ & 0.043 & 0.010 \\
\hline
\end{tabular}

Patients (day 1: day of infection, day 7: 7th day after infection); controls (day 1: first day of hospital admission, day 7: 7th day after admission)

Italic font indicates statistical significance

*Wilcoxon signed rank test

**Mann-Whitney $U$ test forward as a candidate for antibody-based therapy with a potentially promising ability to improve the outcome of S. aureus infection [35]. In a recent study, high levels of specific IgG against IsaA and IsdA among patients with the blistering disease epidermolysis bullosa were reported [36]. Our finding of elevated levels of antibodies against IsaA, IsdA,
IsdH, and $\mathrm{SdrD}$ among bacteremia patients is in agreement with data reported from different geographic regions [17, 20]. This suggests that these antigens could be good targets to be included in a multi-component vaccine.

Many toxins are expressed in vivo during staphylococcal infection, which supports their role in $S$. aureus pathogenesis 
[27]. Among our bacteremia patients, no significant increase in antibodies level against a group of $S$. aureus super antigens in the egc enterotoxin gene cluster (egc) was observed. Corresponding genes for these SAgs were absent among ST239 strains, except for sem. This is in line with the data reported among bacteremia patients previously [17, 20, 37]. Furunculosis patients also developed a low level of antibodies against egc SAgs [38]. In contrast, patients suffering from epidermolysis bullosa showed a higher level of antibodies for this egc SAgs compared with healthy individual controls [36]. On the other hand, genes in the egc cluster are more frequently observed among nasal strains than in strains from infections [39-41]. All these studies indicate that SAgs (egc) may not play critical roles in pathogenesis during staphylococcal bacteremia and furunculosis, whereas they may represent significant factors in epidermolysis bullosa. Although egc and non-egc SAgs are not greatly different with regard to in vitro properties such as $\mathrm{T}$ cell activation, gene regulation, cytokine secretion, or induction of $\mathrm{T}$ cell proliferation [42], diversity in immune response against different types of S. aureus infection emphasizes the fact that they may not be good candidates for vaccine development.

The staphylococcal enterotoxin mainly associated with staphylococcal food poisoning [43-46] is SEA and this is also reported to play an inflammatory role in skin and lung infections $[47,48]$. Antibodies against SEA are commonly observed in healthy individuals $[49,50]$. An association between bacteremia and the presence of genes for sea and tst in the corresponding strains has been reported before [21]. In previous studies among bacteremia patients, no significant differences in $\operatorname{IgG}$ levels against SEA were reported [17, 20], whereas the prevalence of the corresponding gene among causative strains was quite low $(7.6 \%$ and $14.6 \%)$. Here, we observed significantly higher levels of anti-SEA IgG among all bacteremia patients infected with sea gene-positive ST239 MRSA. Our results suggest that SEA could represent a potential active vaccination candidate and that anti-SEA IgG could be used as a passive vaccine.

Staphylococcal superantigen-like proteins (SSLs) are structurally similar to superantigens, but do not share superantigenic activity [51-53]. These proteins interrupt the action of host immune factors: SSL3, for instance, binds to toll-like receptor 2 (TLR2) and interrupts the cytokine production by macrophages $[54,55]$. SSL10 inhibits the classical complement pathway by binding to $\mathrm{Clq}$ and $\mathrm{IgG}$ in general [56]. FC receptor-mediated phagocytosis of neutrophils is interrupted by SSL10 binding to IgG [57]. Gene expression studies of $S$. aureus strains grown in blood revealed no significant upregulation of SSL genes [17, 18]. In our study, no statistically significant difference in the level of $\operatorname{IgG}$ antibodies against SSL1 and SSL10 among bacteremia patients and controls was observed, whereas antibody levels against SSL3, 9, and 10 were significantly reduced among bacteremia patients.

\section{Conclusion}

Clonal isolates of MRSA ST237 induce different immune responses in different patients suffering from bacteremia. However, some antigens induce comparable responses in all patients. We recommend including these antigens (IsaA, IsdA, $\mathrm{IsdH}, \mathrm{SdrD}$, and $\mathrm{HlgB}$ ) in future multi-component vaccines. SEA plays an important role during ST239 MRSA bacteremia and antibody-based therapy using anti-SEA IgG could be a good passive vaccination strategy to improve the outcome of infection. To test the efficacy, additional investigations on the details of the immune response against SEA in other infection models and during in vivo expression are still needed. Our data indicate that as yet ill-defined human factors play an important role in anti-staphylococcal immune regulation.

Acknowledgements We thank Hospital Kuala Lumpur and all the patients who participated in the present study. We take this opportunity to thank and appreciate the participation of doctors, sisters, and staff nurses involved in the study.

Funding This study was funded by the Ministry of Higher Education (MOHE), Malaysia, through the Fundamental Research Grant Scheme (1068 FR) and the Universiti Putra Malaysia.

Compliance with ethical standards The study is in compliance with ethical standards.

Conflicts of interest None to declare except that Alex van Belkum is an employee of bioMérieux, a company developing and selling infectious disease diagnostics.

Ethics approval This study was performed upon approval from the National Institutes of Health for conducting research in the Ministry of Health Malaysia (NMRR-10-488-5953).

Informed consent All subjects were included in the study only if they or their close relatives provided oral consent.

\section{References}

1. Kluytmans JA, Manders MJ, van Bommel E, Verbrugh H (1996) Elimination of nasal carriage of Staphylococcus aureus in hemodialysis patients. Infect Control Hosp Epidemiol 17:793-797

2. Pittet D, Wenzel RP (1995) Nosocomial bloodstream infections: secular trends in rates, mortality, and contribution to total hospital deaths. Arch Intern Med 155(11):1177

3. Hollenbeak CS, Murphy DM, Koenig S, Woodward RS, Dunagan WC, Fraser VJ (2000) The clinical and economic impact of deep chest surgical site infections following coronary artery bypass graft surgery. Chest J 118(2):397-402

4. Rogers DE, Melly M (1965) Speculations on the immunology of staphylococcal infections. Ann N Y Acad Sci 128(1):274-284

5. Oliveira DC, de Lencastre H (2002) Multiplex PCR strategy for rapid identification of structural types and variants of the mec element in methicillin-resistant Staphylococcus aureus. Antimicrob Agents Chemother 46(7):2155-2161

6. Ko KS, Lee J-Y, Suh JY, Oh WS, Peck KR, Lee NY, Song J-H (2005) Distribution of major genotypes among methicillin-resistant 
Staphylococcus aureus clones in Asian countries. J Clin Microbiol 43(1):421-426

7. Ghaznavi-Rad E, Shamsudin MN, Sekawi Z, Khoon LY, Aziz MN, Hamat RA, Othman N, Chong PP, van Belkum A, GhasemzadehMoghaddam H (2010) Predominance and emergence of clones of hospital-acquired methicillin-resistant Staphylococcus aureus in Malaysia. J Clin Microbiol 48(3):867-872

8. Sit P, Teh C, Idris N, Sam I, Syed Omar S, Sulaiman H, Thong K, Kamarulzaman A, Ponnampalavanar S (2017) Prevalence of methicillin-resistant Staphylococcus aureus (MRSA) infection and the molecular characteristics of MRSA bacteremia over a two-year period in a tertiary teaching hospital in Malaysia. BMC Infect Dis 17(1):274-277

9. Josefsson E, Hartford O, O'Brien L, Patti JM, Foster T (2001) Protection against experimental Staphylococcus aureus arthritis by vaccination with clumping factor A, a novel virulence determinant. J Infect Dis 184(12):1572-1580

10. Schaffer AC, Solinga RM, Cocchiaro J, Portoles M, Kiser KB, Risley A, Randall SM, Valtulina V, Speziale P, Walsh E (2006) Immunization with Staphylococcus aureus clumping factor B, a major determinant in nasal carriage, reduces nasal colonization in a murine model. Infect Immun 74(4):2145-2153

11. Kuklin NA, Clark DJ, Secore S, Cook J, Cope LD, McNeely T, Noble L, Brown MJ, Zorman JK, Wang XM (2006) A novel Staphylococcus aureus vaccine: iron surface determinant B induces rapid antibody responses in rhesus macaques and specific increased survival in a murine $S$. aureus sepsis model. Infect Immun 74(4): 2215-2223

12. Zhoua H, Xiong Z-Y, Li H-P, Zheng Y-L, Jiang Y-Q (2006) An immunogenicity study of a newly fusion protein Cna-FnBP vaccinated against Staphylococcus aureus infections in a mice model. Vaccine 24(22):4830-4837. https://doi.org/10.1016/j.vaccine.2006. 03.020

13. Stranger-Jones YK, Bae T, Schneewind O (2006) Vaccine assembly from surface proteins of Staphylococcus aureus. Proc Natl Acad Sci 103(45):16942-16947

14. Otto M (2010) Novel targeted immunotherapy approaches for staphylococcal infection. Expert Opin Biol Ther 10(7):1049-1059

15. Proctor RA (2012) Is there a future for a Staphylococcus aureus vaccine? Vaccine 30(19):2921-2927

16. Verkaik NJ, van Wamel WJB, van Belkum A (2011) Immunotherapeutic approaches against Staphylococcus aureus. Immunotherapy 3(9):1063-1073

17. Den Reijer PM, Lemmens-den Toom N, Kant S, Snijders SV, Boelens H, Tavakol M, Verkaik NJ, van Belkum A, Verbrugh HA, van Wamel WJB (2013) Characterization of the humoral immune response during Staphylococcus aureus bacteremia and global gene expression by Staphylococcus aureus in human blood. PLoS One 8(1):e53391

18. Malachowa N, Whitney AR, Kobayashi SD, Sturdevant DE, Kennedy AD, Braughton KR, Shabb DW, Diep BA, Chambers HF, Otto M (2011) Global changes in Staphylococcus aureus gene expression in human blood. PLoS One 6(4):e18617

19. Dryla A, Prustomersky S, Gelbmann D, Hanner M, Bettinger E, Kocsis B, Kustos T, Henics T, Meinke A, Nagy E (2005) Comparison of antibody repertoires against Staphylococcus aureus in healthy individuals and in acutely infected patients. Clin Diagn Lab Immunol 12(3):387-398

20. Verkaik NJ, Boelens HA, de Vogel CP, Tavakol M, Bode LGM, Verbrugh HA, van Belkum A, van Wamel WJB (2010) Heterogeneity of the humoral immune response following Staphylococcus aureus bacteremia. Eur J Clin Microbiol Infect Dis 29(5):509-518

21. Verkaik NJ, de Vogel CP, Boelens HA, Grumann D, Hoogenboezem T, Vink C, Hooijkaas H, Foster TJ, Verbrugh HA, van Belkum A (2009) Anti-staphylococcal humoral immune response in persistent nasal carriers and noncarriers of Staphylococcus aureus. J Infect Dis 199(5):625-632

22. Lindsay JA, Moore CE, Day NP, Peacock SJ, Witney AA, Stabler RA, Husain SE, Butcher PD, Hinds J (2006) Microarrays reveal that each of the ten dominant lineages of Staphylococcus aureus has a unique combination of surface-associated and regulatory genes. $\mathrm{J}$ Bacteriol 188(2):669-676

23. Martineau F, Picard FJ, Roy PH, Ouellette M, Bergeron MG (1998) Species-specific and ubiquitous-DNA-based assays for rapid identification of Staphylococcus aureus. J Clin Microbiol 36(3):618623

24. Shopsin B, Gomez M, Montgomery SO, Smith DH, Waddington M, Dodge DE, Bost DA, Riehman M, Naidich S, Kreiswirth BN (1999) Evaluation of protein a gene polymorphic region DNA sequencing for typing of Staphylococcus aureus strains. J Clin Microbiol 37(11):3556-3563

25. Harmsen D, Claus H, Witte W, Rothganger J, Claus H, Turnwald D, Vogel U (2003) Typing of methicillin-resistant Staphylococcus aureus in a university hospital setting by using novel software for spa repeat determination and database management. J Clin Microbiol 41(12):5442-5448

26. Enright MC, Day NP, Davies CE, Peacock SJ, Spratt BG (2000) Multilocus sequence typing for characterization of methicillinresistant and methicillin-susceptible clones of Staphylococcus aureus. J Clin Microbiol 38:1008-1016

27. Verkaik NJ, Dauwalder O, Antri K, Boubekri I, de Vogel C, Badiou C, Bes M, Vandenesch F, Tazir M, Hooijkaas H (2009) Immunogenicity of toxins during Staphylococcus aureus infection. Clin Infect Dis 50(1):61-68

28. Verkaik N, Brouwer E, Hooijkaas H, van Belkum A, van Wamel W (2008) Comparison of carboxylated and Penta-His microspheres for semi-quantitative measurement of antibody responses to His-tagged proteins. J Immunol Methods 335(1):121-125

29. Martins TB, Augustine NH, Hill HR (2006) Development of a multiplexed fluorescent immunoassay for the quantitation of antibody responses to group A streptococci. J Immunol Methods 316(1):97-106

30. Dinges MM, Orwin PM, Schlievert PM (2000) Exotoxins of Staphylococcus aureus. Clin Microbiol Infect 13(1):16-34

31. Gouaux E, Hobaugh M, Song L (1997) Aapha-Hemolysin, gamma hemolysin, and leukocidin from Staphylococcus aureus: distant in sequence but similar in structure. Protein Sci 6(12):2631-2635

32. Kamio Y, Rahman A, Nariya H, Ozawa T, Izaki K (1993) The two staphylococcal bicomponent toxins, leukocidin and gamma hemolysin, share one component in common. FEBS Lett 321(1):15-18

33. Prevost G, Cribier B, Couppie P, Petiau P, Supersac G, FinckBarbancon V, Monteil H, Piemont Y (1995) Panton-valentine leucocidin and gamma-hemolysin from Staphylococcus aureus ATCC 49775 are encoded by distinct genetic loci and have different biological activities. Infect Immun 63(10):4121-4129

34. Schaffer AC, Lee JC (2008) Vaccination and passive immunisation against Staphylococcus aureus. Int J Antimicrob Agents 32:S71S78

35. Lorenz U, Lorenz B, Schmitter T, Streker K, Erck C, Wehland J, Nickel J, Zimmermann B, Ohlsen K (2011) Functional antibodies targeting IsaA of Staphylococcus aureus augment host immune response and open new perspectives for antibacterial therapy. Antimicrob Agents Chemother 55(1):165-173

36. Van der Kooi-Pol MM, de Vogel CP, Westerhout-Pluister GN, Veenstra-Kyuchukova YK, Duipmans JC, Glasner C, Buist G, Elsinga GS, Westra H, Bonarius HP (2012) High antistaphylococcal antibody titers in patients with epidermolysis bullosa relate to long-term colonization with alternating types of Staphylococcus aureus. J Investig Dermatol 133:847-850

37. Grumann D, Ruotsalainen E, Kolata J, Kuusela P, Järvinen A, Kontinen VP, Broker BM, Holtfreter S (2011) Characterization of 
infecting strains and superantigen-neutralizing antibodies in Staphylococcus aureus bacteremia. Clin Vaccine Immunol 18(3): 487-493

38. Holtfreter S, Jursa-Kulesza J, Masiuk H, Verkaik NJ, de Vogel C, Kolata J, Nowosiad M, Steil L, van Wamel W, van Belkum A (2011) Antibody responses in furunculosis patients vaccinated with autologous formalin-killed Staphylococcus aureus. Eur J Clin Microbiol Infect Dis 30(6):707-717

39. Ferry T, Thomas D, Genestier A-L, Bes ML, Lina G, Vandenesch F, Etienne J (2005) Comparative prevalence of superantigen genes in Staphylococcus aureus isolates causing sepsis with and without septic shock. Clin Infect Dis 41(6):771-777

40. Jarraud S, Alix Peyrat M, Lim A, Tristan A, Bes ML, Mougel C, Etienne J, Vandenesch F, Bonneville M, Lina G (2001) egc, a highly prevalent operon of enterotoxin gene, forms a putative nursery of superantigens in Staphylococcus aureus. J Immunol 166:669-677

41. Lina G, Bohach GA, Nair SP, Hiramatsu K, Jouvin-Marche E, Mariuzza R (2004) Standard nomenclature for the superantigens expressed by staphylococcus. J Infect Dis 189(12):2334-2336

42. Grumann D, Scharf SS, Holtfreter S, Kohler C, Steil L, Engelmann S, Hecker M, Volker U, Broker BM (2008) Immune cell activation by enterotoxin gene cluster (egc)-encoded and non-egc superantigens from Staphylococcus aureus. J Immunol 181(7): 5054-5061

43. Betley MJ, Mekalanos JJ (1985) Staphylococcal enterotoxin a is encoded by phage. Science 229(4709):185-187

44. Cha JO, Lee JK, Jung YH, Yoo JI, Park YK, Kim BS, Lee YS (2006) Molecular analysis of Staphylococcus aureus isolates associated with staphylococcal food poisoning in South Korea. J Appl Microbiol 101(4):864-871

45. Kerouanton A, Hennekinne JA, Letertre C, Petit L, Chesneau O, Brisabois A, De Buyser ML (2007) Characterization of Staphylococcus aureus strains associated with food poisoning outbreaks in France. Int J Food Microbiol 115(3):369-375

46. Wieneke AA, Roberts D, Gilbert RJ (1993) Staphylococcal food poisoning in the United Kingdom, 1969-90. Epidemiol Infect 110(03):519-531

47. O’Brien GJ, Riddell G, Elborn JS, Ennis M, Skibinski G (2006) Staphylococcus aureus enterotoxins induce IL-8 secretion by human nasal epithelial cells. Respir Res 7:115

48. Cho J, Cho S-Y, Lee KS (2009) Roles of SEA-expressing Staphylococcus aureus, isolated from an atopic dermatitis patient, on expressions of human ß-defensin-2 and inflammatory cytokines in $\mathrm{HaCaT}$ cells. Int J Mol Med 23:331-335

49. Holtfreter S, Bauer K, Thomas D, Feig C, Lorenz V, Roschack K, Friebe E, Selleng K, Lovenich S, Greve T (2004) egc-encoded superantigens from Staphylococcus aureus are neutralized by human sera much less efficiently than are classical staphylococcal enterotoxins or toxic shock syndrome toxin. Infect Immun 72(7): 4061-4071

50. Kunstmann G, Schroder E, Hasbach H, Pulverer G (1989) Immune response to toxic-shock-syndrome toxin-1 (TSST-1) and to staphylococcal enterotoxins A, B and C in Staphylococcus aureus infections. Zentralbl Bakteriol 271(4):486-492

51. Al-Shangiti AM, Naylor CE, Nair SP, Briggs DC, Henderson B, Chain BM (2004) Structural relationships and cellular tropism of staphylococcal superantigen-like proteins. Infect Immun 72(7): 4261-4270

52. Arcus VL, Langley R, Proft T, Fraser JD, Baker EN (2002) The three-dimensional structure of a superantigen-like protein, SET3, from a pathogenicity island of the Staphylococcus aureus genome. J Biol Chem 277(35):32274-32281

53. Fitzgerald JR, Reid SD, Ruotsalainen E, Tripp TJ, Liu M, Cole R, Kuusela P, Schlievert PM, Järvinen A, Musser JM (2003) Genome diversification in Staphylococcus aureus: molecular evolution of a highly variable chromosomal region encoding the staphylococcal exotoxin-like family of proteins. Infect Immun 71(5):2827-2838

54. Bardoel BW, Vos R, Bouman T, Aerts PC, Bestebroer J, Huizinga EG, Brondijk THC, van Strijp JAG, de Haas CJC (2012) Evasion of toll-like receptor 2 activation by staphylococcal superantigen-like protein 3. J Mol Med 90(10):1109-1120

55. Yokoyama R, Itoh S, Kamoshida G, Takii T, Fujii S, Tsuji T, Onozaki K (2012) Staphylococcal superantigen-like protein 3 binds to the Toll-like receptor 2 extracellular domain and inhibits cytokine production induced by Staphylococcus aureus, cell wall component, or lipopeptides in murine macrophages. Infect Immun 80(8): 2816-2825

56. Itoh S, Hamada E, Kamoshida G, Yokoyama R, Takii T, Onozaki K, Tsuji T (2010) Staphylococcal superantigen-like protein 10 (SSL10) binds to human immunoglobulin $\mathrm{G}$ (IgG) and inhibits complement activation via the classical pathway. Mol Immunol 47(4):932-938

57. Patel D, Wines BD, Langley RJ, Fraser JD (2010) Specificity of staphylococcal superantigen-like protein 10 toward the human IgG1 Fc domain. J Immunol 184(11):6283-6292 